\title{
Wang procedure: novel minimally invasive procedure for pectus excavatum children with low age
}

\author{
Wenlin Wang*, Chunmei Chen, Weiguang Long, Xuejun Li and Wenjie Wang \\ Department of Cardiothoracic Surgery, Guangdong Second People's Hospital, Xingangzhonglu, Guangzhou, PR China
}

\begin{abstract}
Nuss procedure is not suitable for pectus excavatum patients with low age. In order to get these patients treated satisfactorily, we have designed a novel technique with a new concept. We placed a metal bar above the chest wall depression. After lifting with steel wires, the depression is fixed in the middle of the bar, and the deformed chest wall can be well treated. Our experiences show that this method is suitable for the younger pectus excavatum children.

After Nuss procedure was reported in 1998, most of the pectus excavatum patients received such operation. However, due to the higher recurrence rate and other disadvantages, the procedure is considered unsuitable for low age patients $[1,2]$. Since most of the children are found to have pectus excavatum shortly after birth, and their families want to complete the operation as soon as possible, it is necessary to design a new procedure to get these children treated.
\end{abstract}

\section{Technique}

The patient is located in the supine position. Skin incision is made in the lower part of the anterior chest wall, just in front of the xiphoid process, with a length of about $2 \mathrm{~cm}$. The soft tissues and muscles are separated to the bottom of the depression just above the surface of the bone structure, then the separation range is enlarged and two tunnels for metal bar are made on both sides of the chest wall. The xiphoid process is exposed and split in the midline longitudinally. After proper traction, the posterior structures behind the sternum are dissociated, and the connection between the diaphragm and the bottom of the sternum is cut off. Three steel wires were sutured through the deformed part of depression, two passing through the costal arches, and one passing through the lower end of the sternum, with the deformed chest wall being completely lifted (Figures 1 and 2). A metal bar is prepared, and its length is about 2 to $3 \mathrm{~cm}$ longer than the distance between the highest points on both sides of the depression. The bar is shaped in

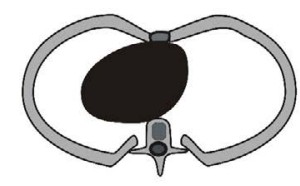

A

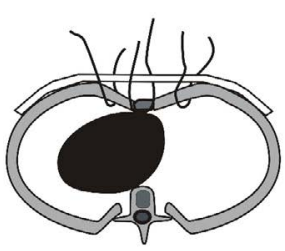

C

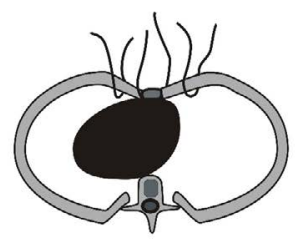

B

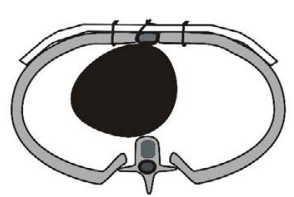

$\mathrm{D}$
Figure 1. (A) Before suturing with steel wires. (B) Three steel wires are sutured successively. (C) The metal bar was inserted into the tennel. (D) Steel wires are pulled up and fixed to the middle part of the bar.

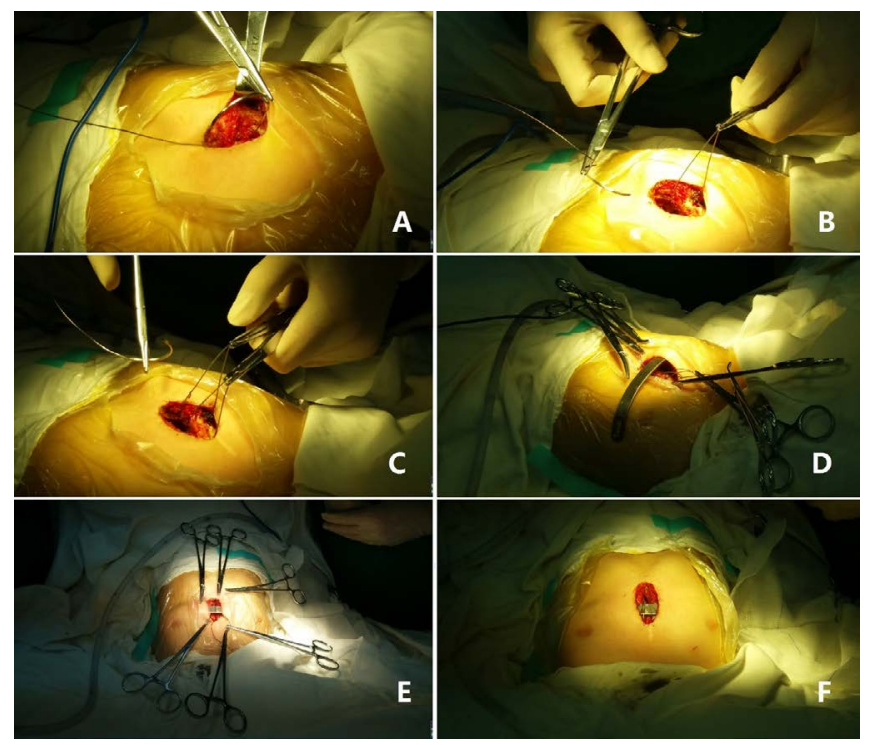

Figure 2. (A), (B) and (C) Three steel wires are sutured successively. (D) and (E) The steel bar was inserted into the tennel. (F) Steel wires are pulled up and fixed to the middle part of the bar.

accordance with the radian of the normal thoracic wall, so that the shape of the bar is equivalent to the patient's chest. Put one end of the bar into the tunnel on one side of the chest wall at first, and then, after reasonable traction, put another end into the opposite tunnel. When

${ }^{\star}$ Correspondence to: Wenlin Wang, Institutions and affiliations: Department of Cardiothoracic Surgery, Guangdong Second People's Hospital, Xingangzhonglu, Guangzhou, PR China, E-mail: willinew@126.com

Key words: wang procedure, pectus excavatum, minimally invasive operation, low age

Received: April 16, 2018; Accepted: May 14, 2018; Published: May 16, 2018 

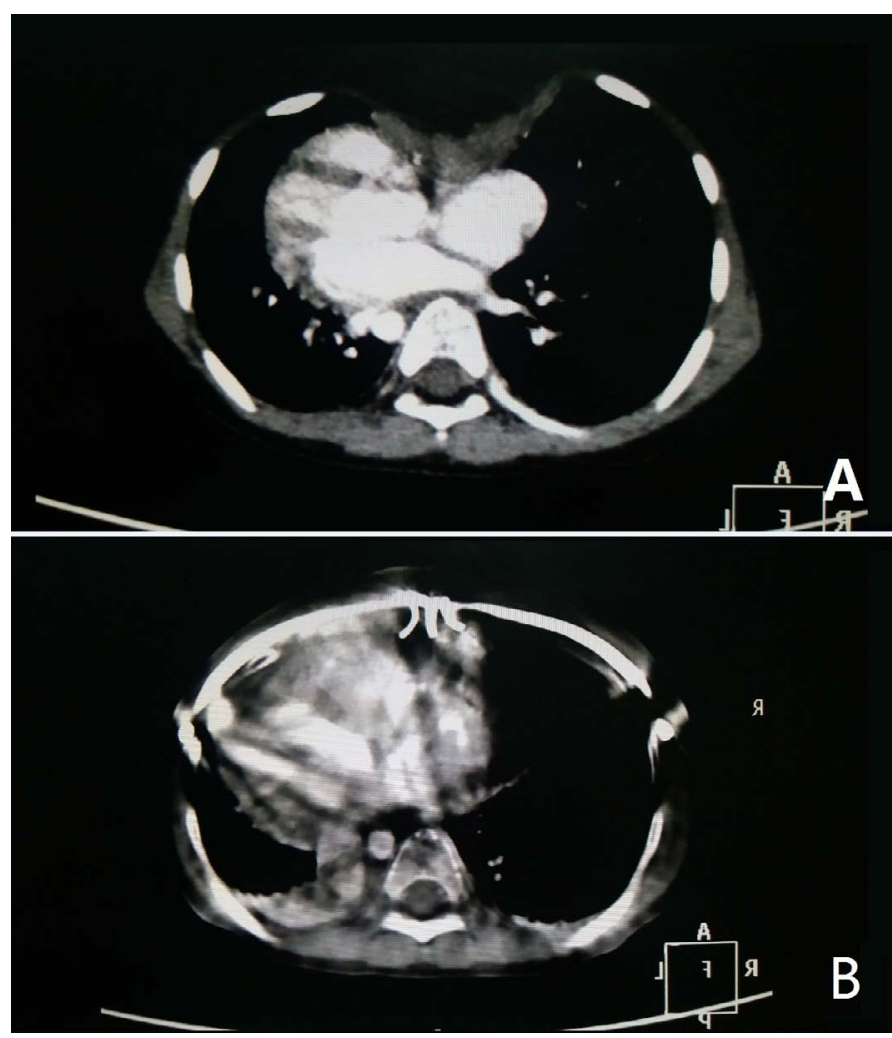

Figure 3. CT images before and after the operation. (A) Pre-operation. (B) Post operation. The depression of the anterior chest wall was raised and pressed to the steel bar, and the appearance of the chest wall was restored to normal.

the position of the bar is adjusted satisfactorily, the 3 steel wires that have been sutured are pulled up and fixed to the middle part of the bar firmly, so that the depressed chest wall can be lifted up completely (Figures 1-3). The muscles and soft tissues surrounding the incision were sutured to embed the bar. After the skin is sutured, the operation is completed.

\section{Comment}

Pectus excavatum is a common deformity of the chest wall, and its surgical treatment has more than 100 years of history. Since the trauma of early techniques was large, few people used them any longer when the minimally invasive Nuss procedure appeared. Nuss procedure is a useful surgical method that has lots of advantages compared with the traditional techniques. However, because of the particularity of its design, there are some defects in it, and one of the most important defects is the restriction of age. It is generally believed that Nuss procedure is not suitable for children of low age. The main reason is worrying about the high recurrence rate caused by early operation $[1,2]$, but in practice, other reasons cannot be ignored.
In Nuss procedure, when the metal bar is rotated to support the depression, the bar needs a hard and rigid fulcrum, otherwise it will not work effectively, and even worse, the chest wall beneath the bar will be pressed into a new depression. In younger patients, their bones were not completely ossified, and is not suitable to act as the fulcrum, therefore, their operation will be greatly affected. In addition, the development of chest of younger patients had relatively rapid speed. If the metal bar is put into their body, as its size and shape do not change with age, the growth of the chest will be restrained. It is because of these reasons that Nuss procedure is rarely used in low age patients, especially under 3 years old [3].

According to our experience, early surgery does not increase the recurrence rate. The key factor is to have the right method of operation, and not only focus on the Nuss procedure.

In our method, we used a completely different method to complete the treatment. We put the metal bar on the surface of the bone structures and complete the correction by pulling the depression with steel wires. In children of low age, because their chest wall is very soft, a slight pull can eliminate the depression, which makes the operation very effective and simple. The metal bar in our procedure is placed on the surface of several ribs, instead of being confined to only one rib like Nuss procedure, therefore, the stress will be dispersed, and the chest wall will not be depressed obviously. In addition, our bar is relatively short, just beyond the edges of the depression, which make the bar is more like "floating" above the chest wall, unlike "clamping" in the the chest wall in Nuss procedure. With this special short bar, the possibility of the constraint of chest wall is greatly reduced, and our procedure is more reasonable for younger children operation than Nuss procedure.

Above all, our procedure has other advantages: (1) only one small incision is needed in our procedure, which will reduce the related trauma correspondingly; (2) our procedure will not pass the pleural cavity, and the related complications will be eliminated completely; (3) the placement of the bar will not be close to the heart; thus, the risk of operation will be greatly reduced.

Until now, we have used this technique in 21 pectus excavatum patients with low age. Our experiences show that this procedure is a simple, safe and effective technique (Figure 3 ). We have made a followup for more than 1 years, and no obvious postoperative complications, including obvious recurrence, have been found. However, due to the limited clinical experience, and relatively short follow-up, its long-term effect remains to be further observed.

\section{References}

1. Nuss D, Kelly RE (2010) Indications and Technique of Nuss Procedure for Pectus Excavatum. Thorc Surg Clin 20: 583-97. [Crossref]

2. Hebra A, Calder BW, Lesher A (2016) Minimally invasive repair of pectus excavatum. J Vis Surg 2: 73. [Crossref]

3. Johnson WR, Fedor D, Singhal S (2014) Systematic review of surgical treatment techniques for adult and pediatric patients with pectus excavatum. $J$ Cardiothorac Surg 9: 25. [Crossref] 